FACTA UNIVERSITATIS

Series: Mechanical Engineering Vol. 17, N ${ }^{\mathrm{o}} 2,2019$, pp. 169 - 180

https://doi.org/10.22190/FUME190312023B

Original scientific paper

\title{
ON THE PROBLEM OF STRAIN LOCALIZATION AND FRACTURE SITE PREDICTION IN MATERIALS WITH IRREGULAR GEOMETRY OF INTERFACES
}

\author{
Ruslan Balokhonov, Varvara Romanova \\ Institute of Strength Physics and Materials Science, SB RAS, Tomsk, Russia
}

\begin{abstract}
The interfacial mechanisms of the stress-strain localization in non-homogeneous media are investigated, using a steel substrate - iron boride coating composition subjected to tension as an example. A dynamic boundary-value problem in a plane-strain formulation is solved numerically by the finite-difference method. The curvilinear substrate-coating interface geometry is assigned explicitly in calculations and is in agreement with experiment. Constitutive relations accounting for an elastic-plastic response of the isotropically-hardened substrate and for a brittle fracture of the coating are employed. Three stages of the plastic strain localization in the steel substrate are found to occur due to the irregular interface geometry. Distributions of the stress concentration regions in the coating are shown to be different at different stages. The stress concentration in the coating is demonstrated to increase nonlinearly during the third stage. The location of fracture is found to depend on the strength of the coating.
\end{abstract}

Key Words: Mesomechanics, Interfaces, Plasticity, Fracture, Coated Materials

\section{INTRODUCTION}

Stress-strain localization phenomena have been much studied both experimentally and theoretically (see, for instance, [1-3]) and may be due to different physical processes operating at different scale levels.

At the microlevel, dislocations, slip bands, dislocation cells, fragmented structures, etc., are formed in single crystals and in grains of polycrystals. It is $a b$ initio geometry associated with the lattice discreteness which is responsible for the stress-strain localization in this case.

The macroscopic stress-strain localization may be due to the geometry of mechanically contacting elements or specimens even at the elastic stage of loading [4], for instance, due to the

Received March 12, 2019 / Accepted June 24, 2019

Corresponding author: Ruslan Balokhonov

Affiliation: Institute of Strength Physics and Materials Science, SB RAS, 634055 Tomsk, Russia

E-mail: rusy@ispms.tsc.ru 
shape of indenters [5]. A prominent example of plastic strain localization is a familiar necking phenomenon. In simulating this deformation instability observed in homogeneous specimens under uniaxial loading, the form of the plastic potential [6] generally plays a decisive role. Changes in the yield surface under plastic deformation result from competitive strain hardening and softening processes and can be described with the use of a phenomenological approach or physically-based dislocation theories. For example, in [7], strain localization occurs where the strain hardening coefficient reaches its critical value, and in [8] the softening rate is a decisive factor for the onset of localization. Another famous example of the macroscopic strain localization is the propagation of Luders bands. A physical substantiation of the process is found to be of microscopic origin and is attributed to the dynamic strain aging effects. A gradual involvement of local regions of the material in plastic deformation is associated with the mechanism for dislocation locking by interstitial atom atmospheres. The dislocations pinned in this way require extra energy to tear away and continue in motion. A macroscopic collective effect of the dislocation behavior gives rise to formation and slow motion of a localized plastic deformation front, resulting in a yield drop and a yield plateau in the macroscopic stress-strain curve. A large number of experimental (see, e.g., [9-12]) and theoretical studies [12-16] have dealt with the Luders band and Portevin-Le Chatelier effects.

The mesoscopic stress-strain localization is associated with different interfaces between microstructural components: interfaces between a matrix and reinforcing particles in composite materials, different phases of alloys, a coating or a hardened surface layers and base material, grain or pore boundaries, etc. The curvilinear interface is a major factor responsible for the occurrence of rotational deformation modes and local geometrical stress concentrations. A number of papers was devoted to analytical modeling of, e.g., interface curvature effects [17]. An abundance of investigations deals with numerical simulations where an explicit account is taken of the material microstructure (see, e.g., [18-21]). Different constitutive models have been developed to describe the mechanical response of individual microstructural components. The goal of these and related investigations is to study and gain insight into the mechanisms for and the special features of the stress-strain localization in the vicinity of interfaces and to examine their effects on the macroscopic mechanical properties of materials.

In this work, we investigate a special feature of the mesoscopic stress-strain localization associated with a self-consistent evolution of stress-strain patterns in a nonhomogeneous material. Particular emphasis is placed on the fact that the geometrical stress concentration regions appearing in the vicinity of curvilinear interfaces and initially randomly distributed over the bulk of the material tend to interact with each other. Due to an ongoing competition of strain hardening and stress relaxation during plastic deformation, a system of stress concentration regions evolves under the action of external forces, approaching equilibrium. The evolution gives rise to stress redistribution. New regions favorable for a rapid stress growth are formed, and there are other regions where the previously formed stress concentrations are suppressed. This is not believed to be a stochastic or spontaneous process; rather, it is predetermined from the outset and governed by the characteristics of the non-homogeneous medium. Three controlling factors of critical importance are involved here: (1) the difference in the mechanical properties of contacting materials (reinforcing particles, matrices, coatings, substrates, interlayers, polycrystalline grains, etc.), (2) the interfacial curvature, and (3) the parameters of external loading. The challenges of mathematical modeling and numerical simulations are to 
reveal and investigate the individual effect of each of the factors on the location of maximum stress-strain concentration regions in which fracture occurs at a certain instant of loading time.

The aim of the present paper is to investigate the mesoscopic stress-strain localization phenomenon, using uniaxial loading of a coated material as an example. The major factor responsible for the evolution of a deformable system such as this is a curvilinear coatingsubstrate interface. Special attention is given to the influence of the plastic strain localization in the substrate on the location and evolution of maximum stress concentrations in the elastic-brittle coating.

\section{STATEMENT OF THE PROBLEM}

Let us consider the microstructure of a steel specimen subjected to surface hardening by a diffusion borating technique. This technology enables high-strength coatings with needleshaped high-curvature profiles to be produced (Fig. 1a). The technique is used for repairing and strengthening the surface of machine parts and structural components. The coated steels take on increased surface hardness and high resistance to impact loads, friction and abrasive wear. In our earlier works [15, 22], we have examined microstructures of this type in some detail. Studies were made on different aspects of deformation and fracture of coated materials, including Luders band propagation, coating thickness and strain rate effects.
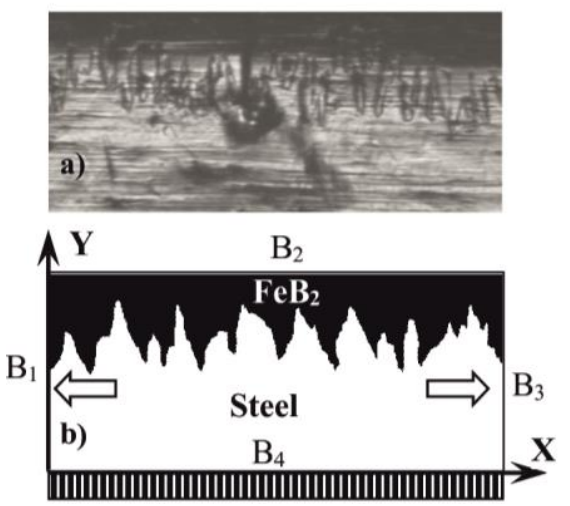

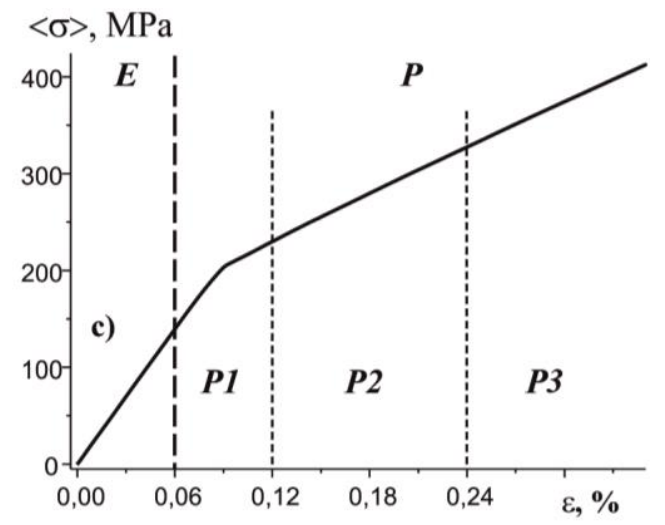

Fig. 1 Experimental [24] (a) and model microstructures (b) and a calculated microscopic stress-strain curve (c) for a specimen with a curvilinear coating-substrate interface

In this contribution, particular emphasis is placed on an analysis of the evolution of stress concentration in the vicinity of the interface. A total system of equations for simulating the deformation of a coated material includes mass and momentum conservation laws, strain relations and constitutive equations describing the material response [15, 23]. In the case at hand, the use is made of models for elastic-plastic behavior of the steel substrate and for a brittle fracture of the iron-boride coating. A dynamic boundary-value problem in a plane strain formulation is solved numerically by the finite difference method [22, 23, 25].

The boundary conditions for the left- $\mathrm{B}_{1}$ and right-hand surfaces $\mathrm{B}_{3}$ of the computational domain simulate uniaxial tension of the coated material along the $\mathrm{X}$ direction, while those 
for the top B2 and bottom surfaces B4 correspond to the conditions for free surface and symmetry, respectively, (Fig. 1b).

The steel substrate exhibits an elastic-plastic behavior. The plastic flow rule $\dot{\varepsilon}_{\mathrm{ij}}^{\mathrm{p}}=\dot{\lambda} \mathrm{S}_{\mathrm{ij}}$ is associated with a yield surface:

$$
\sigma_{\mathrm{eq}}=\phi\left(\varepsilon_{\mathrm{eq}}^{\mathrm{p}}\right)
$$

where $\sigma_{\text {eq }}$ is the equivalent stress and $\varepsilon^{\mathrm{p}}{ }_{\text {eq }}$ is the cumulative equivalent plastic strain, $\varepsilon^{\mathrm{p}}{ }_{\mathrm{ij}}$ is the plastic strain tensor components and $\lambda$ is a scalar parameter equal to zero in the elastic region.

The following function satisfying the experimental data is used to describe the isotropic hardening of mild steel:

$$
\phi\left(\varepsilon_{e q}^{p}\right)=\sigma_{s}-\left(\sigma_{s}-\sigma_{0}\right) \exp \left(-\varepsilon_{e q}^{p} / \varepsilon_{r}^{p}\right)
$$

where $\sigma_{\mathrm{s}}$ and $\sigma_{0}$ are the ultimate strength and the initial yield point, respectively, and $\varepsilon_{\mathrm{r}}^{\mathrm{p}}$ is a characteristic value of the equivalent plastic strain.

Cracking of the coating was examined, using a maximum distortion energy criterion. In $[15,22,23]$ the foregoing modified fracture criterion employed in combination with an explicit account of the material microstructure is shown to provide an adequate direction of crack propagation in brittle compounds. According to the criterion, fracture occurs where the equivalent stress reaches limiting values $\mathrm{C}_{\text {ten }}$ or $\mathrm{C}_{\text {com }}$ depending on the type of the stressed state (tension or compression) found in a given local region:

$$
\sigma_{\mathrm{eq}}=\left\{\begin{array}{l}
\mathrm{C}_{\mathrm{ten}}, \text { for } \varepsilon_{\mathrm{kk}}>0 \\
\mathrm{C}_{\mathrm{com}}, \text { for } \varepsilon_{\mathrm{kk}}<0
\end{array}\right.
$$

Here $\mathrm{C}_{\text {ten }}$ and $\mathrm{C}_{\mathrm{com}}$ are the strength of iron boride under tension and compression.

The fracture criterion given by Eq. (3) accounts for the following factors. A local coating region subjected to tension $\left(\varepsilon_{\mathrm{kk}}>0\right)$ fails where the local equivalent stress reaches a value $\mathrm{C}_{\text {ten. }}$. It is assumed that for the failed coating regions both the deviator $\mathrm{S}_{\mathrm{ij}}=0$ and the pressure $\mathrm{P}=-\mathrm{K} \varepsilon_{\mathrm{kk}}=0$. For compressive regions $\left(\varepsilon_{\mathrm{kk}}<0\right)$, the limiting fracture surface in stress space is restricted by $\mathrm{C}_{\text {com. }}$. In this case, the failed coating material offers no resistance to shear alone $\left(S_{\mathrm{ij}}=0\right)$.

The mechanical properties of steel substrate and iron boride coating are listed in Table 1. Note that $K$ and $\mu$ denote the respective bulk and shear moduli.

Table 1 The mechanical properties of the steel and iron-boride [24, 26]

\begin{tabular}{lccccccc}
\hline & $\mathrm{K}$, & $\mu$, & $\sigma_{\mathrm{s}}$, & $\sigma_{0}$, & $\varepsilon_{\mathrm{r}}^{\mathrm{p}}$ & $\mathrm{C}_{\text {ten }}$, & $\mathrm{C}_{\text {com, }}$ \\
& $\mathrm{GPa}$ & $\mathrm{GPa}$ & $\mathrm{MPa}$ & $\mathrm{MPa}$ & & $\mathrm{GPa}$ & $\mathrm{GPa}$ \\
\hline Substrate & 133 & 80 & 395 & 174 & 0,093 & - & - \\
Coating & 200 & 140 & - & - & - & 1.1 & 4 \\
\hline
\end{tabular}




\section{COMPUTATIONAL RESUlts}

Figure 1c depicts a homogenized stress-strain curve for a coated material subjected to tension. The stress is calculated as an equivalent stress averaged over the computational domain: $\langle\sigma\rangle=\sum_{\mathrm{k}=1, \mathrm{~N}} \sigma_{\mathrm{eq}}^{\mathrm{k}} \mathrm{s}^{\mathrm{k}} / \sum_{\mathrm{k}=1, \mathrm{~N}} \mathrm{~s}^{\mathrm{k}}$, where $\mathrm{N}$ is the number of computational cells and $\mathrm{s}^{\mathrm{k}}$ is the k-th cell area. Strain $\varepsilon$ corresponds to the elongation of the computational domain along the X-axis: $\varepsilon=\left(\mathrm{L}-\mathrm{L}_{0}\right) / \mathrm{L}_{0}$, where $\mathrm{L}_{0}$ and $\mathrm{L}$ are the initial and the current specimen lengths.

There are two main deformation stages in the stress-strain curve: $E$ and $P$ denote elasticity and plasticity, respectively. At the elastic stage, both the steel substrate and the boride coating are strained elastically. Because of the difference in the elastic moduli between the coating and the substrate, stress and elastic strain distributions exhibit a nonuniform pattern. Local regions of stress concentration occur in the coating material near the coating-substrate interface (Fig. 2, regions 1-9). The stress value in these regions is dictated by the local interfacial geometry. At the plastic stage, the coating still exhibits an elastic behavior, whereas the substrate deforms plastically.

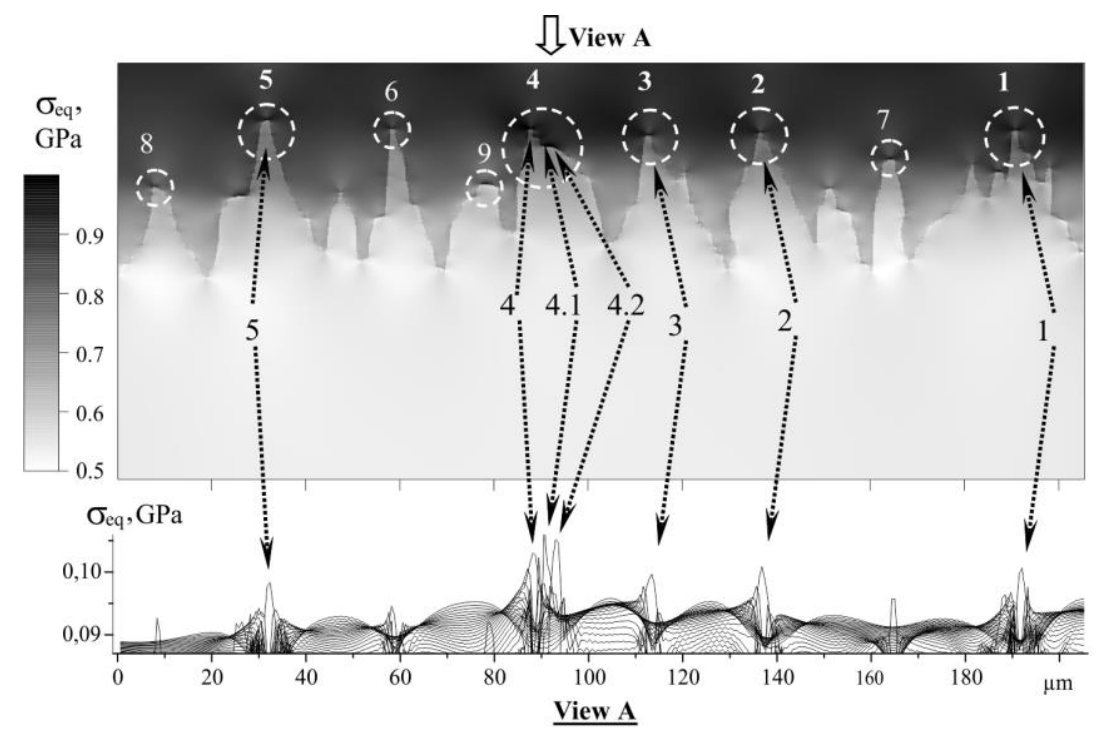

Fig. 2 Equivalent stress concentrations in the vicinity of the coating-substrate interface at the elastic deformation stage: the total strain of the coated material $\varepsilon$ is $0.03 \%$ (see Fig. 1c)

Let us examine the evolution of the most powerful stress concentrations (peaks 1-5 in Fig. 2) formed in the elastic coating. Figs. 3 and 4 show the initial evolution period including stage $E$ and substages $P 1$ and $P 2$. The plots in Fig. 3a show the manner in which the equivalent stresses at the peaks $\sigma_{\text {eq }}{ }^{i}$ vary with the specimen elongation. By and large, the growth rate is different for different peaks (1-5), but the stresses are seen to exhibit a nearly linear growth. It should be pointed out that both in Fig. 3a and in the stress-strain curve (Fig. 1c) the deformation stages and substages are not clearly visible. However, they are quite discernible in the case where the relative stress level is examined (Fig. 3b) 
$\delta \sigma_{\text {eq }}^{\mathrm{i}}=\frac{\sigma_{\mathrm{eq}}^{\mathrm{i}}-\left\langle\sigma_{\mathrm{eq}}^{\mathrm{i}}\right\rangle}{\left\langle\sigma_{\mathrm{eq}}^{\mathrm{i}}\right\rangle}$, where $\left\langle\sigma_{\mathrm{eq}}^{\mathrm{i}}\right\rangle=\frac{1}{5} \sum_{\mathrm{i}=1}^{5} \sigma_{\mathrm{eq}}^{\mathrm{i}}$ is the magnitude of the equivalent stress averaged over the 5 peaks.

As seen from Fig. $3 b, \delta \sigma_{\text {eq }}$ is nearly constant during elastic deformation of the coated steel. This means that the stress values at peaks 1-5 increase at the same rate, and the stress patterns remain qualitatively unchanged (compare Figs. 2 and 4a). At stage $E$, there is an average stress level in regions $1-3$, whereas the stress values $\sigma_{\text {eq }}^{4}$ and $\sigma_{\text {eq }}^{5}$ are by $5 \%$ higher and by $5 \%$ lower than $\left\langle\sigma_{\text {eq }}^{\mathrm{i}}\right\rangle$, respectively (see view A in Figs. 2 and $3 \mathrm{~b}$ ). Three stress peaks are formed in region 4 of the highest stress concentration. A maximum equivalent stress is found to be in local region 4.1 (Fig. 2). The elastic stage continues until the total strain amounts to $0.06 \%$ (Figs. $3 \mathrm{~b}$ and 1c).

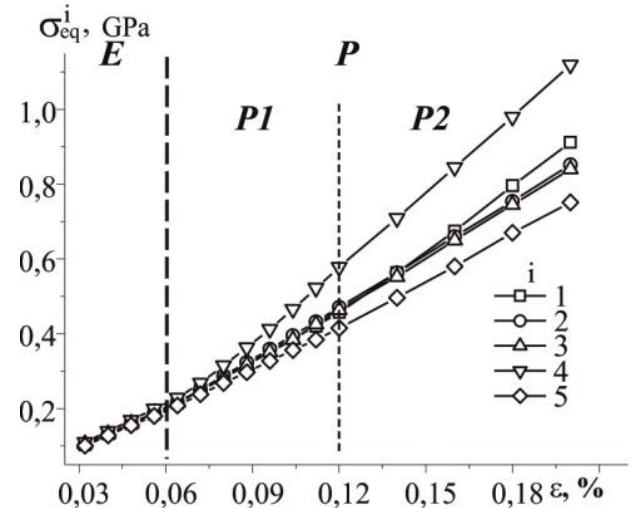

a)

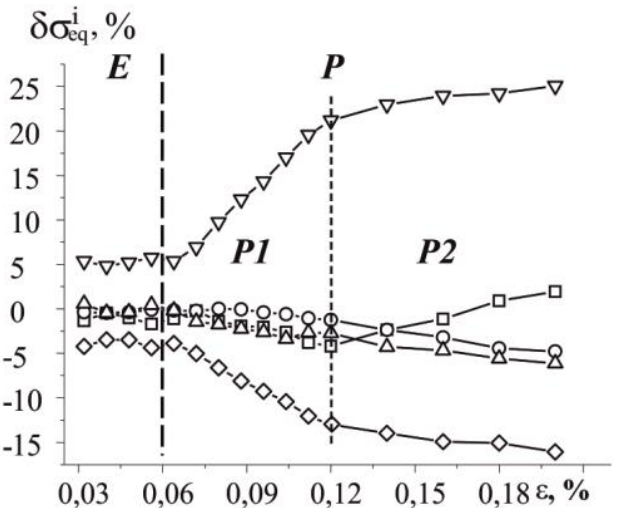

b)

Fig. 3 Evolution of maximum equivalent stresses (see Fig. 2) in regions 1-5 (a) and their deviations from the average value $\left\langle\sigma_{\text {eq }}^{\mathrm{i}}\right\rangle$ (b) during elastic stage 1 and plastic substages $\mathrm{P} 1$ and $\mathrm{P} 2$ of the coated material deformation

On further loading, the steel substrate deforms plastically, with substages $P 1$ and $P 2$ being well-pronounced in strain ranges of $0.06-0.12$ and $0.12-0.24 \%$, respectively (Fig. 3b). At substage $P 1$, the steepest rise in the maximum stress is observed in region 4 (Figs. $3 \mathrm{~b}$ and 4). Remarkably, out of 3 peaks located in this region the equivalent stress increases where its magnitude is at a minimum at the elastic stage (Fig. 2, arrows 4). To the contrary, in regions 4.1 and 4.2, the local stress rate slows down (Fig. 4). The same conclusion suggests itself for region 5 , where the stress-strain localization is suppressed (Figs. 3 and 4). In regions 1-3, the stress growth rate is still close to the average level $\left\langle\sigma_{\text {eq }}^{\mathrm{i}}\right\rangle$, slowing down late at substage $P 1$.

At substage $P 2$, the qualitative evolution pattern is, on the whole, retained: stresses $\delta \sigma_{\text {eq }}^{2}$ and $\delta \sigma_{\text {eq }}^{3}$ continue to decrease at a higher rate, whereas $\delta \sigma_{\text {eq }}^{4}$ and $\delta \sigma_{\text {eq }}^{5}$ continue to increase and decrease, respectively, but the rate of change is lower (Fig. 3b) than at substage $P l$. The only exception is that a characteristic feature inherent to this deformation stage is a change in the 
slope of the curve for stress peak 1 . This means that the stress-strain localization in region 1 is enhanced (Fig. 3b, boxes).

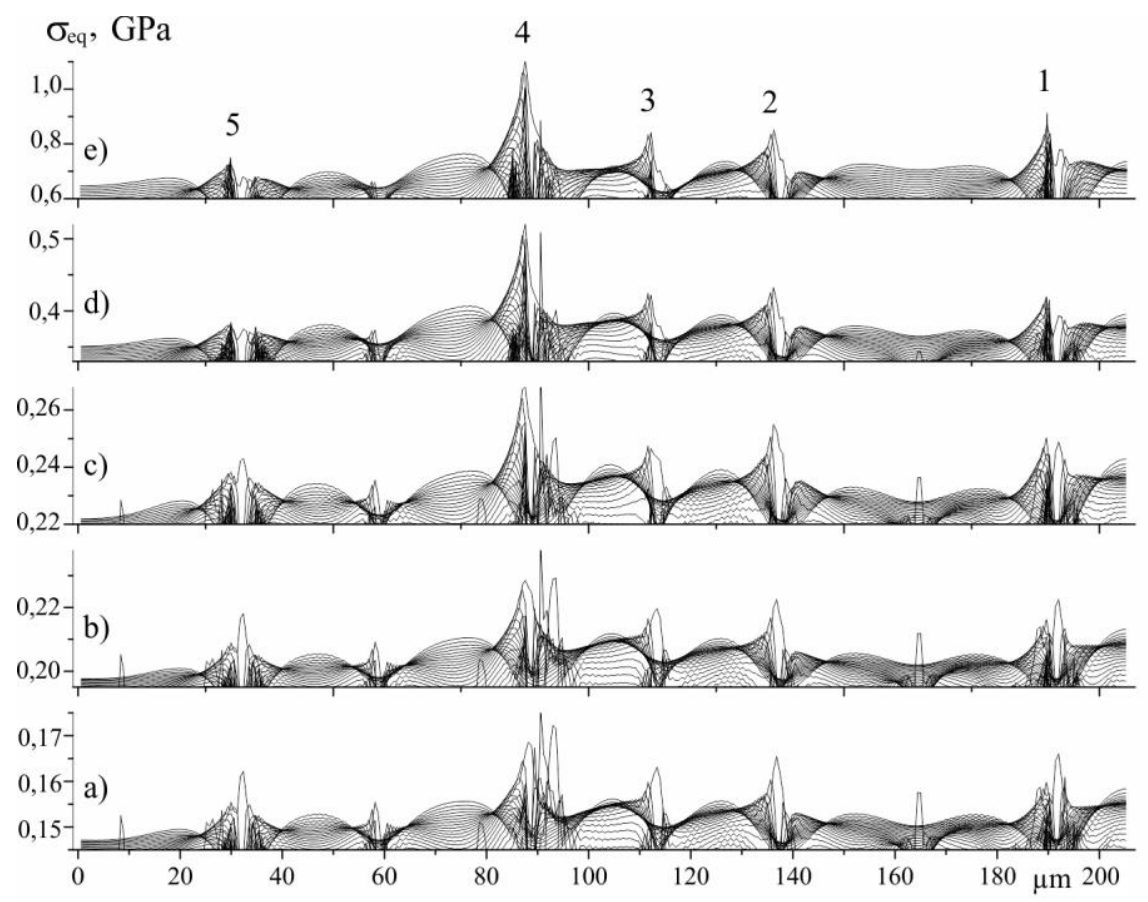

Fig. 4 Evolution of the equivalent stress pattern at substages $P 1$ and $P 2$ of the coated material deformation. The total strain $\varepsilon=0.046$ (a), 0.06 (b), 0.07 (c), 0.11 (d), and $0.2 \%$ (e) (see Figs. 1c and 3). Video data file "fig4.avi" is available online

The simulation results were analyzed to show that the substages of the stress peak evolution in the boride coating were associated with a specific character of plastic strain localization in the steel substrate (Figs. 5 and 6). At substage $P 1$, the plastic strains nucleating near the interface cover the substrate material in a step-by-step manner. First, plastic shear strains arise in the steel material at the roots of boride teeth (Fig. 5a) and propagate deep into the material, filling up the space between the teeth (Fig. $5 b-d$ ), with the major part of the substrate material being in the elastic state. Then localized shear bands start to form in the bulk (Fig. $5 \mathrm{e}-\mathrm{f}$ ). The bands originate near the interface asperities (mainly at the tooth humps) and are localized in conjugate directions at an angle of $\approx 45$ degrees to the tensile direction, causing an abrupt change in the slope of the macroscopic stress-strain curve (Fig. 1c). Substage $P 1$ is over when most of the substrate material transforms into a plastic state, with the band system being formed completely.

At substage $P 2$ (Fig. 5f, Fig. 6a), the band distribution changes but only slightly, while the plastic strain localization in the bands is enhanced to form a clearly visible shear band system. This ordering of the shear bands is due to the competing stress relaxation and strain hardening processes in local regions of the substrate material. 
To summarize the foregoing simulation results for the initial evolution period, the stress growth rate in local near-interface regions of the elastic iron boride coating is constant at stage $E$, linearly increases in region 4 and slows down in region 5 at substages $P 1$ and $P 2$ due to plastic strain nucleation and shear band formation in the steel substrate.

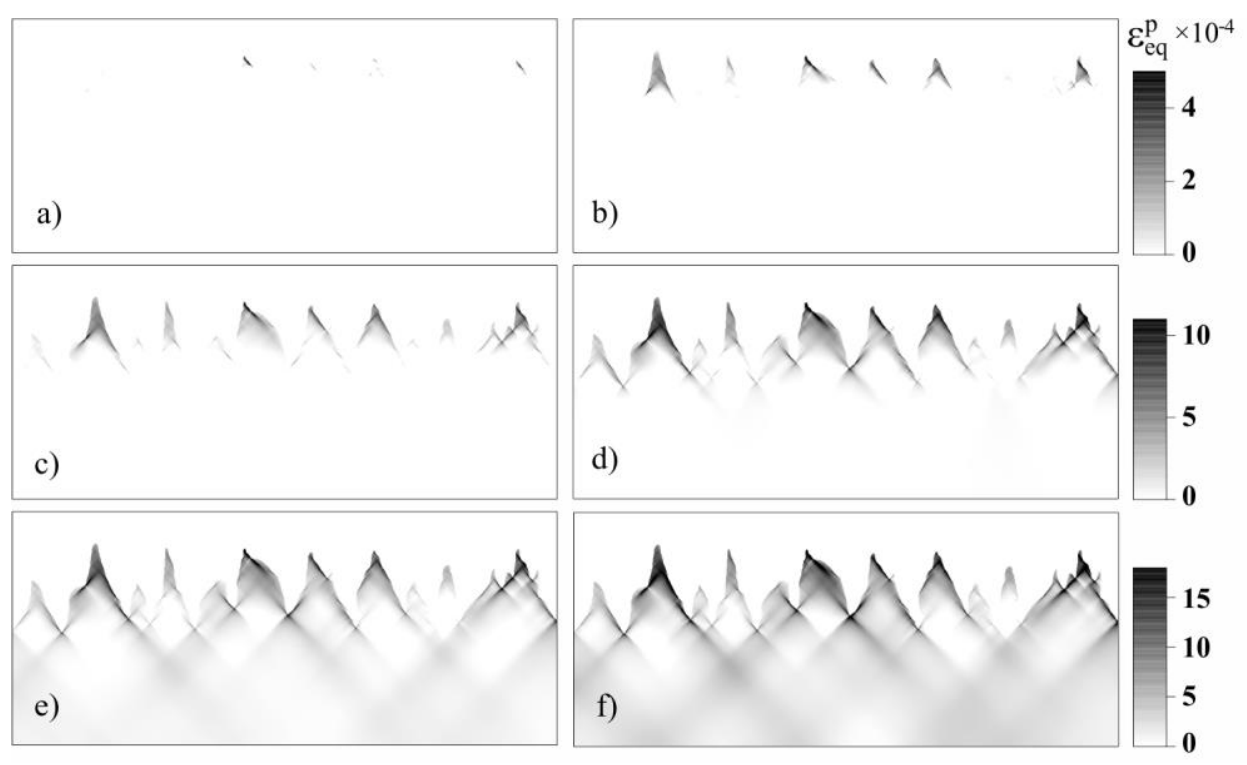

Fig. 5 Equivalent plastic strain patterns for total strain $\varepsilon=0.06$ (a), 0.07(b), 0.08 (c), 0.09 (d), 0.1 , (e) and $0.11 \%$ (f); substage $P 1$

Quite a different evolution pattern is seen at substage $P 3$ characterized by nonlinear stress-strain localization in the vicinity of the coating-substrate interface. Much as at substages $P 1$ and $P 2$, this is due to the special features of the plastic strain localization in the substrate material. Analysis of the obtained results shows that the conjugate shear bands intersecting at an angle of 45 degree are smeared at substage $P 3$. The plastic strain localization mechanism in the steel substrate changes: due to a decrease in the local strain hardening coefficient in near-interface regions, the localization in the shear bands (Fig. 6a) gives way to the localization along the coating-substrate interface alone (Fig. 6b). As a consequence, the evolution of the stress concentration acquires a reverse pattern. A fast nonlinear rise of the stress peak is observed in region 5 (Figs. 7 and 8), i.e., in the region where the stress-strain localization was hitherto suppressed (Figs. 3 and 4). At the same time, the seemingly most powerful stress peak in region 4 that dominated at previous stages is seen to weaken rapidly. At a certain instant of loading time (see inverted triangles in Fig. 8b), the stress growth rate for $\sigma_{\text {eq }}^{4}$ becomes the lowest among the $\sigma_{\text {eq }}^{\mathrm{i}}$ rates, being inferior in magnitude even to the rates of initially less-developed $\sigma_{\text {eq }}^{2}$ and $\sigma_{\text {eq. }}^{3}$. Equivalent stress $\sigma_{\text {eq }}^{1}$ increases steadily at an average rate of $\left\langle\sigma_{\text {eq }}^{\mathrm{i}}\right\rangle$ and will exceed $\sigma^{4}$ eq on further loading when the curves 1 and 4 in Fig. $8 \mathrm{~b}$ intersect. 


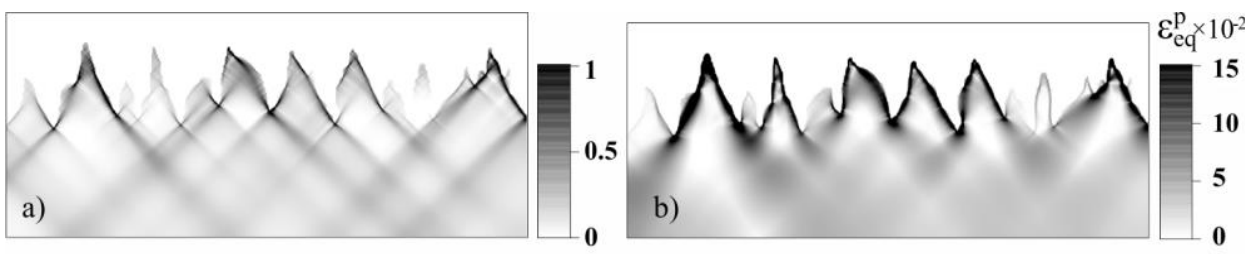

Fig. 6 Equivalent plastic strains for the total strain $\varepsilon=0.24$ (a) and $2.41 \%$ (b); substage $P 3$

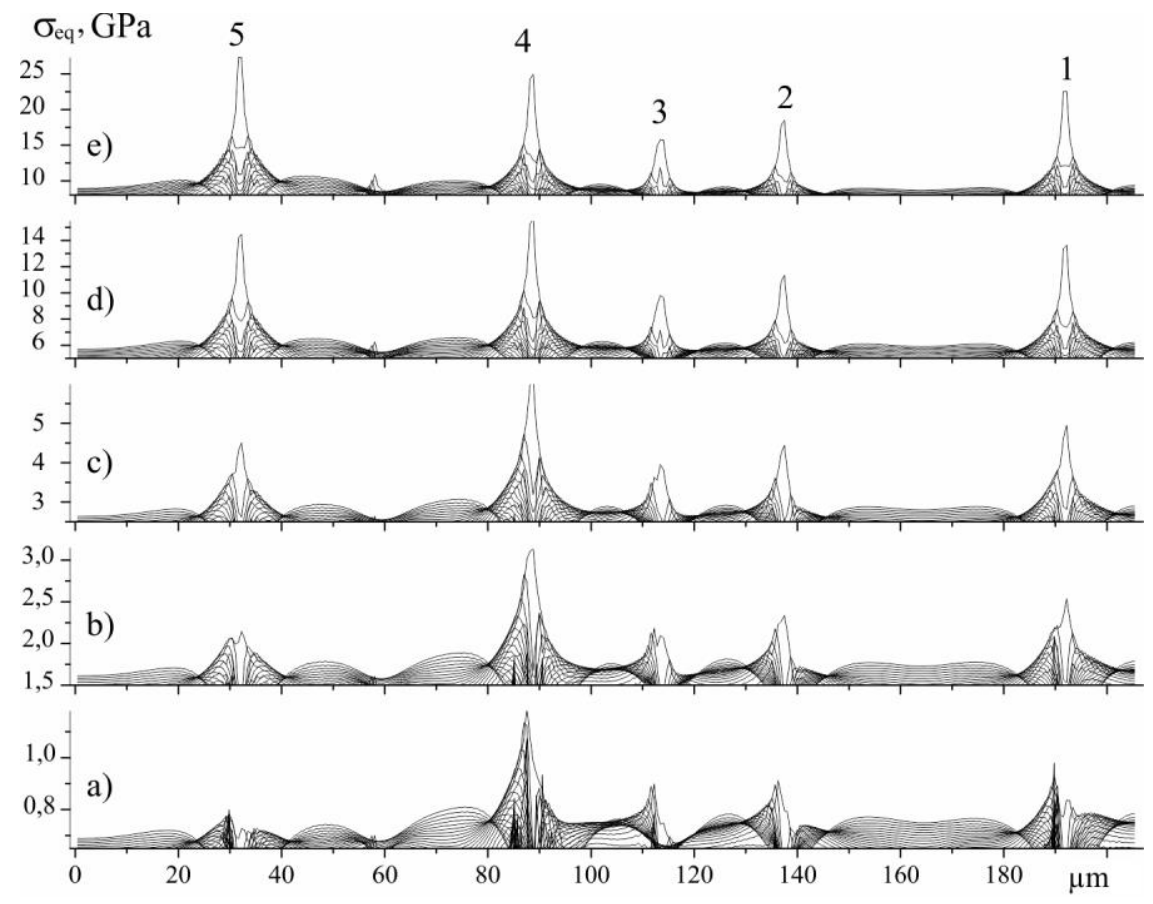

Fig. 7 Evolution of the equivalent stress pattern at substage $P 3$ of the coated material deformation. The total strain $\varepsilon=0.24$ (a), 0.51(b), 0.81(c), 1.71(d) and $2.61 \%$ (e).

Unlike the quasi-linear stress growth in regions 1-4, the dynamics of the stress-strain localization in region 5 is nonlinear (Fig. 8a) with the highest local stress growth rate (Fig. 8b). It is in this region where the local interfacial geometry is seen to be the most favorable for the plastic strain localization along two neighboring boride tooth sides. In other words, these sides are oriented in the directions lying most closely to those of maximum tangential stresses. This fact enhances the straining in region 5 and causes the most rapid increase in the stress concentration in the coating as compared to regions 1-4. 

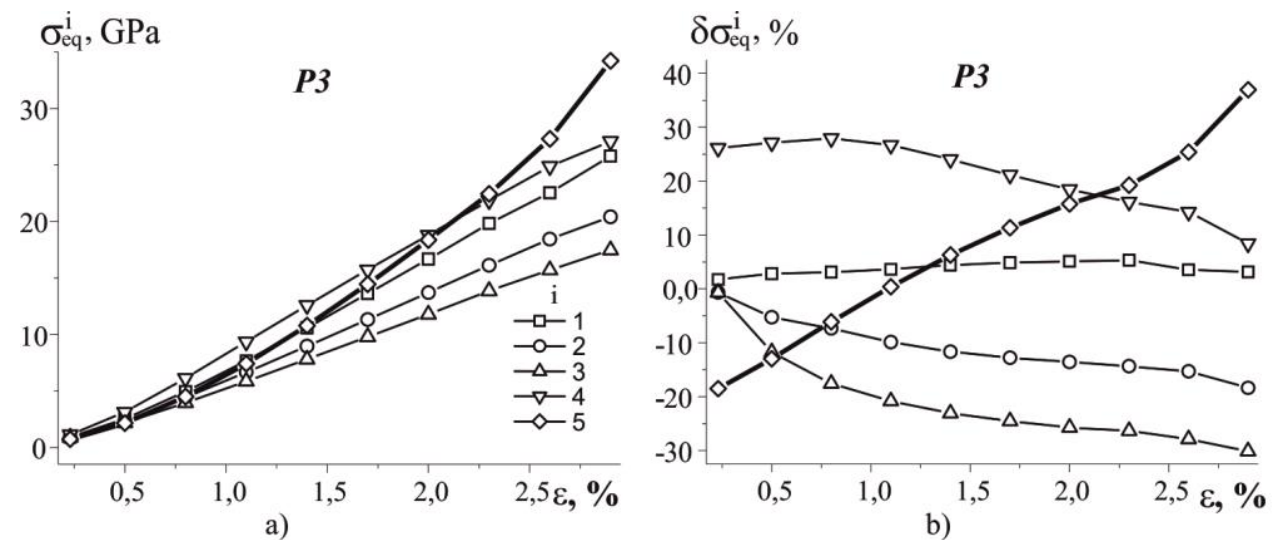

Fig. 8 Evolution of maximum equivalent stresses (see Figs. 2 and 8) in regions 1-5 (a) and their deviations from the average value $\left\langle\sigma_{\text {eq }}^{\mathrm{i}}\right\rangle$ (b) at substage $P 3$ of the coated material deformation
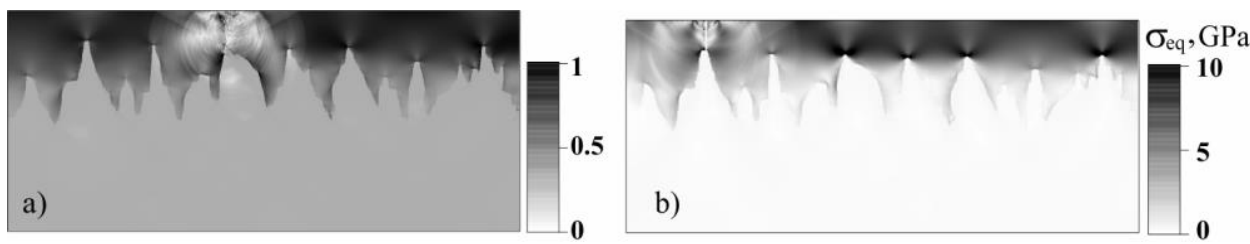

Fig. 9 Equivalent stress patterns showing the fracture localization for varying coating strength of 1.1 (a) and $21 \mathrm{GPa}(\mathrm{b})$

Thus the maximum stress concentration developing in the coating along the coatingsubstrate interface is found at different points depending on the stage of the coated material deformation: maximum equivalent stresses are observed in regions $4.1,4$ and 5 at stage $E$, substages $P 1-P 2$ and substage $P 3$, respectively. This means that the fracture site can vary with the deformation stage. In other words, for a given microstructural geometry and mechanical properties of the constituent materials, the coating strength determines the fracture location. The numerical simulation results illustrating this conclusion are presented in Fig. 9. Two calculations for varying coating strength were performed to show the difference in the fracture patterns. A crack in the coating originates in a near-interface region of a maximum stress concentration and propagates perpendicular to the tensile direction towards the free surface that corresponds to the experiment (Fig. 1a). For a low-strength coating, cracking occurs in region 4 (Fig. 9a) at substage $P 2$ for the total strain $\varepsilon=0.2 \%$ (Figs. 3 and 4), whereas a high-strength coating fails (Fig. 9b) late at substage $P 3$ for $\varepsilon=2.2 \%$ under the maximum local stress conditions in region 5 (Figs. 7 and 8). 


\section{CONCLUSION}

A mesomechanical analysis of the stress-strain localization and fracture in iron boride coating - steel substrate composition under uniaxial tension has been performed. A dynamic boundary-value problem was solved numerically by the finite-difference method. The elastic-brittle and elastic-plastic properties were assigned for the coating and substrate materials, respectively. A curvilinear coating-substrate interface corresponded to the configuration found experimentally and was accounted for explicitly in calculations.

Due to the difference in the elastic moduli between steel and boride, local regions of the stress-strain localization were shown to arise along the interface even at the elastic stage of the coated material deformation. The stress concentration in the coating regions depends on the local interfacial geometry. Redistribution of the stress concentration peaks discussed in this paper is not found to have occurred at the elastic stage.

Three stages of plastic deformation in the steel substrate have been revealed. The first stage corresponds to the nucleation of plastic strains in local near-interface regions and their propagation deep into the steel material to involve the internal regions in plastic flow. The second stage represents formation of well-defined shear bands oriented at an angle of 45 degrees to the loading direction and plastic strain localization in the bands. At the third stage, the shear bands are smeared, and the plastic flow is localized along the curvilinear interface alone.

The stage-by-stage change in the mechanisms for the plastic strain localization in the steel substrate is responsible for the stress concentration redistribution in the near-interface coating regions, the location of maximum equivalent stress is found to vary with the deformation stages. Consequently, the fracture localization depending on the critical equivalent stress was found to be affected by the coating strength. Notably, the stress-strain localization at the third stage was found to develop in a nonlinear manner and was seen to occur where it was suppressed at previous stages.

The evolution of the stress-strain localization suggests the general conclusions that are not limited to the particular case of the coated material considered in this work. To prove or reject this assumption, further investigations are warranted into the effects of the mechanical properties of constituents, interfacial geometry and loading conditions on a special character of the nonlinear stress-strain localization in composite materials.

Acknowledgements: This work is supported by the Fundamental Research Program of the State Academies of Sciences for 2013-2020, line III.23.

\section{REFERENCES}

1. Vinogradov, A., Estrin, Y., 2018, Analytical and numerical approaches to modelling severe plastic deformation, Progress in Materials Science, 95, pp.172-242.

2. Antolovich, S.D., Armstrong, R.W., 2014, Plastic strain localization in metals: origins and consequences, Progress in Materials Science, 59, pp.1-160.

3. Lunt, D., Xu, X., Busolo, T., Quinta da Fonseca, J., Preuss, M., 2018, Quantification of strain localisation in a bimodal two-phase titanium alloy, Scripta Materialia, 145, pp. 45-49.

4. Popov, V.L., 2010, Contact mechanics and friction: physical principles and foundations, Springer, Berlin

5. Li, Q., Popov, V.L., 2016, Indentation of flat-ended and tapered indenters with polygonal crosssections, Facta Universitatis-Series Mechanical Engineering, 14(3), pp. 241-249. 
6. Becker, R., Needleman, A., 1986, Effect of yield surface curvature on necking and failure in porous plastic solids, J. Appl. Mech., 53, pp. 491-499.

7. Dequiedt, J.L., 2013, Localization in heterogeneous materials: A variational approach and its application to polycrystalline solids, International Journal of Plasticity, 48, pp. 92-110.

8. Xue, L., 2010, Localization conditions and diffused necking for damage plastic solids, Engineering Fracture Mechanics, 77, pp. 1275-1297.

9. Beukel, A.V.D., Kocks, U.F., 1982, The strain dependence of static and dynamic strain-aging, Acta Metall. Mater, 30, pp. 1027-1034.

10. Neuhäuser, H., Hampel, A., 1993, Observation of Luders bands in single crystals, Scripta Metallurgica et Materialia, 29, pp. 1151-7.

11. Casarotto, L., Tutsch, R., Ritter, R., Weidenmüller, J., Ziegenbein, A., Klose, F., Neuhäuser, H., 2003, Propagation of deformation bands investigated by laser scanning extensometry, Computational Materials Science, 26, pp. 210-218.

12. Anjabin, N., Karimi Taheri, A., Kim, H.S., 2013, Simulation and experimental analyses of dynamic strain aging of a supersaturated age hardenable aluminum alloy, Materials Science \& Engineering A, 585, pp. 165-173.

13. McCormick, P.G., Ling, C.P., 1995, Numerical modeling of the Portevin-Le Chatelier effect, Acta Metall. Mater, 43, pp. 1969-1977.

14. Hähner, P., Rizzi, E., 2003, On the kinematics of Portevin-Le Chatelier bands: theoretical and numerical modeling, Acta Materialia, 51, pp. 3385-3397.

15. Balokhonov, R.R., Romanova, V.A., Martynov, S.A., Schwab, E.A., 2013, Simulation of Deformation and Fracture of Coated Material with Account for Propagation of a Lïders-Chernov Band in the Steel Substrate, Physical Mesomechanics, 16(2), pp. 133-140.

16. Balokhonov, R.R., Romanova, V.A., Schmauder, S., Makarov, P.V., 2003, Simulation of meso-macro dynamic behavior using steel as an example, Computational Materials Science 28, pp. 505-511.

17. Li, Y., Ortiz, Ch., Boyce, M.C., 2013, A generalized mechanical model for suture interfaces of arbitrary geometry, Journal of the Mechanics and Physics of Solids, 61, pp. 1144-1167.

18. Panin, V.E., (Eds.), 1998, Physical mesomechanics of heterogeneous media and computer-aided design of materials, Cambridge International Science Publishing, Cambridge.

19. Needleman, A., 2000, Computational mechanics at the mesoscale, Acta Materialia, 48, pp. 105-124.

20. Schmauder, S., Schäfer, I., 2016, Multiscale Materials Modeling: Approaches to Full Multiscaling, Walter de Gruyter GmbH \& Co KG, Berlin.

21. Balokhonov, R.R., Romanova, V.A., Panin, A.V., Kazachenok, M.S., Martynov, S.A., 2018, Strain Localization in Titanium with a Modified Surface Layer, Physical Mesomechanics, 21(1), pp. 32-42.

22. Balokhonov, R., Zinoviev, A., Romanova, A., Zinovieva, O., 2016, The computational micromechanics of materials with porous ceramic coatings, Meccanica 51(2), pp. 415-428.

23. Balokhonov, R.R., Romanova, V.A., Schmauder, S., Schwab, E.A., 2012, Mesoscale analysis of deformation and fracture in coated materials, Computational Materials Science 64, pp. 306-311.

24. Koval, A.V., Panin, S.V., 2000, Mesoscale deformation and cracking of surface-hardened low carbon steel, Theoretical and Applied Fracture Mechanics 34, pp. 117-121.

25. Richtmyer, R.D., Morton, K.W., 1967, Difference methods for initial-value problems, Interscience publishers, John Wiley \& Sons, New York - London - Sydney.

26. Grigorieva, I.S., Meilihova, E.Z., (Eds.), 1991, Physical values, Reference book, Energoatomizdat, Moscow. 